\title{
An Electron Transfer Approach to the Preparation of Highly Functionalized Anthraquinones
}

\section{Abdelouahab Beziane, Thierry Terme and Patrice Vanelle*}

Laboratoire de Chimie Organique Pharmaceutique, CNRS-UMR 6517, Faculté de Pharmacie, 27 Bd Jean Moulin, 13385 Marseille Cx 5, France

* To whom correspondence should be addressed; E-mail: patrice.vanelle@pharmacie.univ-mrs.fr.

Received: 24 August 2004 / Accepted: 23 September 2004 / Published: 31 January 2005

\begin{abstract}
A series of highly functionalized quinones was prepared by an original reaction of 2,3-bis(chloromethyl)-1,4-dimethoxyanthraquinone (6) with various nitronate anions under electron transfer reaction conditions.
\end{abstract}

Keywords: Anthraquinones, electron transfer, anthracyclines.

\section{Introduction}

The efficacy of anthracycline antibiotics such as daunomycin and adriamycin in the treatment of a variety of human malignancies has stimulated a continued interest in the synthesis of this class of antitumor agents [1-3]. Unfortunately, their clinical use is limited by a number of problems, including intrinsic and acquired drug resistance and dose-dependent cardiomyopathy. Considerable efforts have been devoted to develop new structurally modified anthracyclines with an improved antineoplastic activity and a low cardiotoxicity.

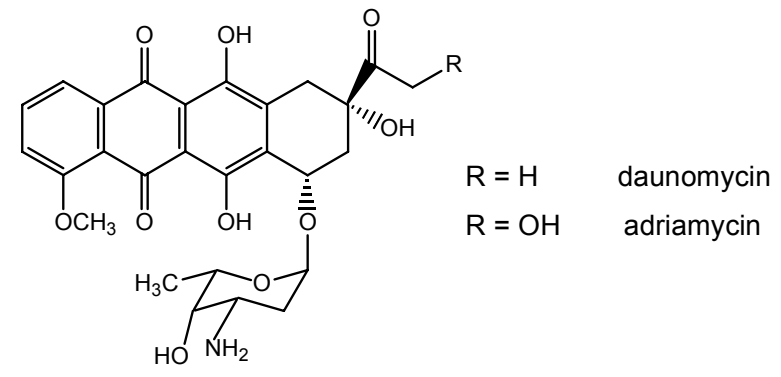


As bioreductible alkylating agents, quinones constitute potential substrates for the radical nucleophilic substitution $\left(\mathrm{S}_{\mathrm{RN}} 1\right)$ reaction [4]. In connection with our program directed toward the synthesis of new nitroheterocyclic or quinonic bioreductible alkylating agents using electron transfer methodology [5], we report herein the preparation of 2,3-bis(chloromethyl)-1,4dimethoxyanthraquinone (6) via a four-step synthesis from phthalic anhydride (1) and 2,3-dimethyl1,4-hydroquinone (2) and the study of its reactivity with various anions to afford new highly functionalized anthraquinonic derivatives.

\section{Results and Discussion}

1,4-Dihydroxy-2,3-dimethylanthraquinone (3) was isolated in $80 \%$ yield after condensation of phthalic anhydride (1) and 2,3-dimethyl-1,4-hydroquinone (2) in the presence of aluminium chloride [6]. The methylation of compound 3 with dimethylsulfate (DMS) in acetone followed by radical bromination using $\mathrm{N}$-bromosuccinimide (NBS) and halogen exchange reaction with lithium chloride furnished the target $\mathbf{6}$ (Scheme 1).

\section{Scheme 1.}


Treated under various electron transfer operating conditions with 2-nitropropane anion, the bischloride 6 led to the bis- $C$-alkylation product 7a (Scheme 2). The best yield of bis- $C$-alkylated product 7a was obtained in phase transfer conditions using dichloromethane as solvent with 5 equivalents of 2nitropropane anion during 20 minutes under inert atmosphere and light catalysis. The reaction of 6 with 2-nitropropane anion, in the optimal conditions, in presence of classical inhibitors ( $p$ dinitrobenzene as radical-anion scavenger, 2,2,6,6-tetramethyl-1-piperidinyloxy or TEMPO as radical trap and $\mathrm{CuCl}_{2}$ ) gave effective inhibition, indicating a classical bis-S $\mathrm{S}_{\mathrm{RN}} 1$ mechanism for the formation of $7 \mathbf{a}$. 


\section{Scheme 2.}

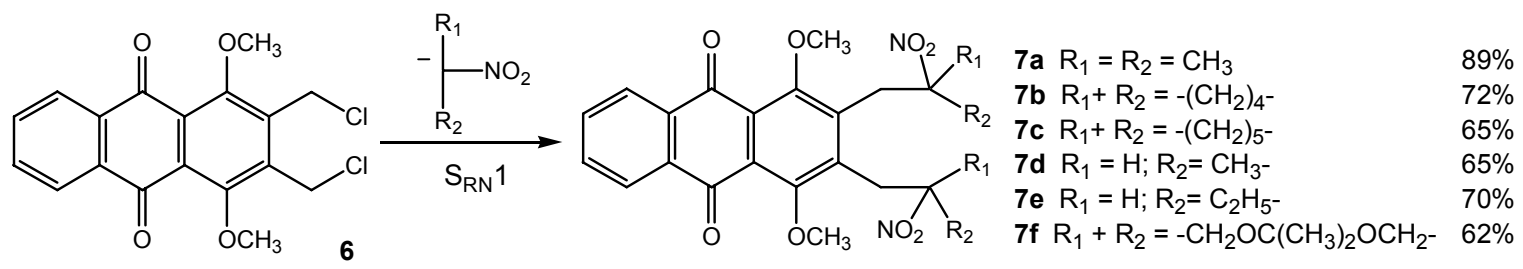

This bis- $\mathrm{S}_{\mathrm{RN}} 1$ reaction was extended with various primary and secondary (cyclic, heterocyclic) nitronate anions (Scheme 2) or $S$-centered anions (Scheme 3) and allowed us to reach at a series of new highly functionalized anthraquinones. These bis- $C$-alkylated compounds $\mathbf{7 a - f}$ are good candidates for the preparation of anthracycline analogs via an annulation reaction after base promoted nitrous acid elimination [7].

\section{Scheme 3.}<smiles>COc1c(CO)c(OC)c2c(c1CO)C(=O)c1ccccc1C2=O</smiles>



The extension of the reactivity to $S$-centered anions was realized from toluenesulfinic acid sodium salt or butanesulfinic acid sodium salt. This latter anion was formed from butane sulfonyl chloride according to the procedure of Liu [8]. These two reactions treated in refluxed DMSO during 2 hours furnished the corresponding bis-S-alkylated compounds $7 \mathbf{g}$ and $\mathbf{7 h}$ in $73 \%$ and $96 \%$ yields, respectively (Scheme 3).

\section{Conclusions}

In conclusion, we have demonstrated in this work that the reaction of a new bis(chloromethyl)anthraquinone $\mathbf{6}$ with nitronate anions proceeded according to an bis-S $\mathrm{S}_{\mathrm{RN}} 1$ mechanism. This procedure permitted us to prepare new highly functionalized anthraquinones in excellent yields. The antiproliferative activities of these compounds are under active investigation.

\section{Experimental}

\section{General}

Melting points were determined on Büchi B-540 and are uncorrected. Elemental analyses were performed by the Centre de Microanalyses of the University of Aix-Marseille 3. Both ${ }^{1} \mathrm{H}-$ and ${ }^{13} \mathrm{C}$-NMR spectra were determined on $\mathrm{CDCl}_{3}$ solutions on a Bruker ARX 200 spectrometer. The ${ }^{1} \mathrm{H}$ chemical shifts are reported as parts per million downfield from tetramethylsilane $\left(\mathrm{Me}_{4} \mathrm{Si}\right)$, and the ${ }^{13} \mathrm{C}$ chemical shifts were referenced to the solvent peak of $\mathrm{CDCl}_{3}(76.9 \mathrm{ppm})$. Silica gel 60 adosrbent (Merck, 230-400 mesh) was used for column chromatography: Thin-layer chromatography was 
performed with silica gel Merck 60F-254 (0.25 mm layer thickness). Compounds 3-5 were prepared according to Kerdesky method [6]. Nitroalkanes were commercially available or easily prepared by oxidation of the corresponding amine with $m$-chloroperbenzoic acid in refluxing 1,2-dichloroethane by Gilbert and Borden's procedure [9]. The nitroheterocyclic nitronate anion was prepared from 2,2dimethyl-5-hydroxymethyl-5-nitro-1,3-dioxane by the previously described method [10]. Toluenesulfinic acid sodium salt was commercially available and butanesulfinic acid sodium salt was prepared from butane sulfonyl chloride according to the Liu procedure [8].

\section{2,3-Bis(chloromethyl)-1,4-dimethoxyanthraquinone (6).}

In a two-necked flask equipped with a drying tube, a solution of 5 (1.7 g, $3.74 \mathrm{mmol})$ and of lithium chloride $(3.25 \mathrm{~g}, 76,67 \mathrm{mmol})$ in anhydrous tetrahydrofuran $(50 \mathrm{~mL})$ was stirred at room temperature under an inert atmosphere for $48 \mathrm{~h}$. Then, dichloromethane $(100 \mathrm{~mL})$ was added. The organic layer was washed twice with water $(50 \mathrm{~mL})$, dried over $\mathrm{MgSO}_{4}$ and removed under reduced pressure. Purification by chromatography on silica gel eluting with dichloromethane and recrystallization from ethanol gave 2,3-bis(chloromethyl)-1,4-dimethoxyanthraquinone (6); mp $140{ }^{\circ} \mathrm{C} .{ }^{1} \mathrm{H}-\mathrm{NMR} \delta$ : 4.04 (s, $\left.6 \mathrm{H}, 2 \mathrm{xOCH}_{3}\right), 4.91\left(\mathrm{~s}, 4 \mathrm{H}, 2 \mathrm{xCH}_{2} \mathrm{Cl}\right), 7.75-7.80(\mathrm{~m}, 2 \mathrm{H}, 2 \mathrm{xAr}-\mathrm{H}), 8.18-8.21(\mathrm{~m}, 2 \mathrm{H}, 2 \mathrm{xAr}-\mathrm{H}) ;{ }^{13} \mathrm{C}-$ NMR $\delta: 35.6\left(2 \mathrm{xCH}_{2} \mathrm{Cl}\right), 63.6\left(2 \mathrm{xOCH}_{3}\right), 126.7(2 \mathrm{xCH}), 127.8(2 \mathrm{xC}), 133.8(2 \mathrm{xC}), 133.9(2 \mathrm{xCH})$, $140.1(2 \times C), 155.7(2 \times C), 182.2(2 \times C=O)$; Anal. Calcd for $\mathrm{C}_{18} \mathrm{H}_{14} \mathrm{Cl}_{2} \mathrm{O}_{4}: \mathrm{C}, 59.20 ; \mathrm{H}, 3.86$. Found: $\mathrm{C}$, $59.23 ; \mathrm{H}, 3.87$.

General procedure for bis- $S_{R N} 1$ reaction with aliphatic and cyclic nitronate anions.

Under a nitrogen atmosphere, a solution of tetrabutylammonium hydroxide ( $40 \%$ in water, $2.1 \mathrm{~mL}$, $2.75 \mathrm{mmol})$ was treated with nitroalkane $(2.75 \mathrm{mmol})$ for $1 \mathrm{~h}$. A solution of 2,3-bis(chloromethyl)-1,4dimethoxyanthraquinone $(6,0.20 \mathrm{~g}, 0.55 \mathrm{mmol})$ in dichloromethane $(20 \mathrm{~mL})$ was then added and the mixture was irradiated with a $300 \mathrm{~W}$ sun lamp for $20 \mathrm{~min}$ at room temperature under an inert atmosphere. The organic layer was separated and the aqueous layer was extracted with dichloromethane $(3 \times 10 \mathrm{~mL})$. The combined organic layers were washed twice with water $(30 \mathrm{~mL})$, dried over $\mathrm{MgSO}_{4}$ and removed under reduced pressure. Purification by chromatography on silica gel eluting with chloroform and recrystallization from ethanol led to the corresponding products $7 \mathbf{a}-\mathbf{f}$.

1,4-Dimethoxy-2,3-bis(2-methyl-2-nitropropyl)anthraquinone (7a): Orange solid, $\mathrm{mp} \quad 168.4^{\circ} \mathrm{C}$ (ethanol); ${ }^{1} \mathrm{H}-\mathrm{NMR} \delta: 1.55$ (s, $\left.12 \mathrm{H}, 4 \mathrm{xCH}_{3}\right), 3.37$ (s, 4H, $\left.\mathrm{CH}_{2}\right), 3.83$ (s, 6H, 2xOCH 3 ), 7.74-7.77 (m, $2 \mathrm{H}, 2 \mathrm{xAr}-\mathrm{H}), 8.17-8.20(\mathrm{~m}, 2 \mathrm{H}, 2 \mathrm{xAr}-\mathrm{H}) ;{ }^{13} \mathrm{C}-\mathrm{NMR} \delta: 26.1\left(4 \mathrm{xCH}_{3}\right), 37.0\left(2 \mathrm{xCH}_{2}\right), 62.0\left(2 \mathrm{xOCH}_{3}\right)$, $88.6\left(2 \mathrm{xCNO}_{2}\right), 125.7(2 \mathrm{xC}), 126.7(2 \mathrm{xCH}), 133.8(2 \mathrm{xCH}), 133.9(2 \mathrm{xC}), 139.4(2 \mathrm{xC}), 156.2(2 \mathrm{xC})$, 182.4 (2xC=O); Anal. Calcd for $\mathrm{C}_{24} \mathrm{H}_{26} \mathrm{~N}_{2} \mathrm{O}_{8}: \mathrm{C}, 61.27 ; \mathrm{H}, 5.57 ; \mathrm{N}, 5.95$. Found: $\mathrm{C}, 61.42 ; \mathrm{H}, 5.60 ; \mathrm{N}$, 5.78 .

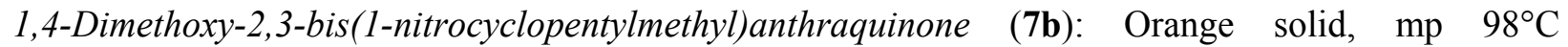
(ethanol); ${ }^{1} \mathrm{H}-\mathrm{NMR} \delta: 1.58-1.69\left(\mathrm{~m}, 8 \mathrm{H}, 4 \mathrm{xCH}_{2}\right), 1.79-1.90\left(\mathrm{~m}, 4 \mathrm{H}, 2 \mathrm{xCH}_{2}\right), 2.49-2.60(\mathrm{~m}, 4 \mathrm{H}$, $\left.2 \mathrm{xCH}_{2}\right), 3.47\left(\mathrm{~s}, 4 \mathrm{H}, 2 \mathrm{xCH}_{2}\right), 3.85\left(\mathrm{~s}, 6 \mathrm{H}, 2 \mathrm{xOCH}_{3}\right), 7.74-7.78(\mathrm{~m}, 2 \mathrm{H}, 2 \mathrm{xAr}-\mathrm{H}), 8.18-8.22(\mathrm{~m}, 2 \mathrm{H}$, 
2xAr-H); ${ }^{13} \mathrm{C}-\mathrm{NMR} \delta: 22.7\left(4 \mathrm{xCH}_{2}\right), 34.7\left(2 \mathrm{xCH}_{2}\right), 36.3\left(4 \mathrm{xCH}_{2}\right), 62.1\left(2 \mathrm{xOCH}_{3}\right), 101.1\left(2 \mathrm{xCNO}_{2}\right)$, $125.9(2 \mathrm{xC}), 126.7(2 \mathrm{xCH}), 133.8(2 \mathrm{xCH}), 134.0(2 \mathrm{xC}), 139.9(2 \times \mathrm{C}), 156.1(2 \times C), 182.5(2 \mathrm{xC}=\mathrm{O})$; Anal. Calcd for $\mathrm{C}_{28} \mathrm{H}_{30} \mathrm{~N}_{2} \mathrm{O}_{8}$ : C, 64.36; H, 5.79; N, 5.36. Found: C, 64.27; H, 5.77; N, 5.17.

1,4-Dimethoxy-2,3-bis(1-nitrocyclohexylmethyl)anthraquinone $\quad(\mathbf{7 c})$ : Yellow solid, $\mathrm{mp} \quad 191^{\circ} \mathrm{C}$ (ethanol); ${ }^{1} \mathrm{H}-\mathrm{NMR} \delta$ : $1.12-1.27\left(\mathrm{~m}, 4 \mathrm{H}, 2 \mathrm{xCH}_{2}\right), 1.59-1.67\left(\mathrm{~m}, 12 \mathrm{H}, 6 \mathrm{xCH}_{2}\right), 2.31-2.45(\mathrm{~m}, 4 \mathrm{H}$, $\left.2 \mathrm{xCH}_{2}\right), 3.18\left(\mathrm{~s}, 4 \mathrm{H}, 2 \mathrm{xCH}_{2}\right), 3.84\left(\mathrm{~s}, 6 \mathrm{H}, 2 \mathrm{xOCH}_{3}\right), 7.73-7.77(\mathrm{~m}, 2 \mathrm{H}, 2 \mathrm{xAr}-\mathrm{H}), 8.17-8.21(\mathrm{~m}, 2 \mathrm{H}$, 2xAr-H); ${ }^{13} \mathrm{C}-\mathrm{NMR} \delta: 22.2\left(6 \mathrm{xCH}_{2}\right), 24.3\left(2 \mathrm{xCH}_{2}\right), 34.2\left(2 \mathrm{xCH}_{2}\right), 37.5\left(2 \mathrm{xCH}_{2}\right), 61.9\left(2 \mathrm{xOCH}_{3}\right), 92.5$ $\left(2 \mathrm{xCNO}_{2}\right), 125.4(2 \mathrm{xC}), 126.6(2 \mathrm{xCH}), 133.7(2 \mathrm{xCH}), 134.0(2 \mathrm{xC}), 139.2(2 \mathrm{xC}), 156.0(2 \mathrm{xC}), 182.5$ $(2 \mathrm{xC}=\mathrm{O})$; Anal. Calcd for $\mathrm{C}_{30} \mathrm{H}_{34} \mathrm{~N}_{2} \mathrm{O}_{8}: \mathrm{C}, 65.44 ; \mathrm{H}, 6.22 ; \mathrm{N}, 5.09$. Found: $\mathrm{C}, 65.48 ; \mathrm{H}, 6.22 ; \mathrm{N}, 4.85$.

1,4-Dimethoxy-2,3-bis(2-nitropropyl)anthraquinone (7d): Orange solid, $\mathrm{mp} 185.9^{\circ} \mathrm{C}$ (ethanol); ${ }^{1} \mathrm{H}-\mathrm{NMR} \delta: 1.58(\mathrm{~d}, 3 \mathrm{H}, J=6.7 \mathrm{~Hz}), 1.68(\mathrm{~d}, 3 \mathrm{H}, J=6.7 \mathrm{~Hz}), 3.19-3.54\left(\mathrm{~m}, 4 \mathrm{H}, 2 \mathrm{xCH}_{2}\right), 3.90(\mathrm{~s}, 6 \mathrm{H}$, $\left.2 \mathrm{xOCH}_{3}\right), 4.91-5.02\left(\mathrm{~m}, 2 \mathrm{H}, 2 \mathrm{xCHNO}_{2}\right), 7.75-7.79(\mathrm{~m}, 2 \mathrm{H}, 2 \mathrm{xAr}-\mathrm{H}), 8.18-8.22(\mathrm{~m}, 2 \mathrm{H}, 2 \mathrm{xAr}-\mathrm{H})$; ${ }^{13} \mathrm{C}-\mathrm{NMR} \delta: 19.5\left(2 \mathrm{xCH}_{3}\right), 33.0\left(2 \mathrm{xCH}_{2}\right), 62.4\left(2 \mathrm{xOCH}_{3}\right), 82.9\left(2 \mathrm{xCHNO}_{2}\right), 125.9(2 \mathrm{xC}), 126.7$ $(2 \mathrm{xCH}), 133.9(2 \mathrm{xCH}), 138.8(2 \mathrm{xC}), 139.4(2 \mathrm{xC}), 156.0(2 \mathrm{xC}), 182.3(2 \times \mathrm{C}=\mathrm{O})$; Anal. Calcd for $\mathrm{C}_{22} \mathrm{H}_{22} \mathrm{~N}_{2} \mathrm{O}_{8}$ : C, 59.73; H, 5.01; N, 6.33. Found: C, 59.74; H, 4.84; N, 6.39.

1,4-Dimethoxy-2,3-bis(2-nitrobutyl)anthraquinone (7e): Orange solid, mp $145.8{ }^{\circ} \mathrm{C}$ (ethanol); ${ }^{1} \mathrm{H}$ NMR $\delta: 1.00(\mathrm{t}, 3 \mathrm{H}, J=7.4 \mathrm{~Hz}), 1.05(\mathrm{t}, 3 \mathrm{H}, J=7.4 \mathrm{~Hz}), 1.81-2.18\left(\mathrm{~m}, 4 \mathrm{H}, 2 \mathrm{xCH}_{2}\right), 3.22-3.45(\mathrm{~m}$, $\left.4 \mathrm{H}, 2 \mathrm{xCH}_{2}\right), 3.90\left(\mathrm{~s}, 6 \mathrm{H}, 2 \mathrm{xOCH}_{3}\right), 4.74-4.83\left(\mathrm{~m}, 2 \mathrm{H}, 2 \mathrm{CHNO}_{2}\right), 7.75-7.78(\mathrm{~m}, 2 \mathrm{H}, 2 \mathrm{xAr}-\mathrm{H}), 8.18-$ $8.21(\mathrm{~m}, 2 \mathrm{H}, 2 \mathrm{xAr}-\mathrm{H}) ;{ }^{13} \mathrm{C} \mathrm{NMR} \delta: 10.2\left(2 \mathrm{xCH}_{3}\right), 27.9\left(2 \mathrm{xCH}_{2}\right), 31.4\left(2 \mathrm{xCH}_{2}\right), 62.3\left(2 \mathrm{xOCH}_{3}\right), 89.3$ $\left(2 \mathrm{xCHNO}_{2}\right), 125.7$ (2xC), $126.7(2 \mathrm{xCH}), 133.8(2 \mathrm{xC}), 133.9$ (2xCH), 139.5 (2xC), 155.9 (2xC), 182.4 $(2 \mathrm{xC}=\mathrm{O})$; Anal. Calcd for $\mathrm{C}_{24} \mathrm{H}_{26} \mathrm{~N}_{2} \mathrm{O}_{8}$ : C, 61.27; H, 5.57; N, 5.95. Found: C, 61.15; H, 5.74; N, 5.95.

2,3-Bis(2,2-dimethyl-5-nitro[1,3] dioxan-5-ylmethyl)-1,4-dimethoxyanthraquinone (7f): Yellow solid, mp $215^{\circ} \mathrm{C}$ (ethanol); ${ }^{1} \mathrm{H}-\mathrm{NMR} \delta: 1.35\left(\mathrm{~s}, 6 \mathrm{H}, 2 \mathrm{xCH}_{3}\right), 1.50\left(\mathrm{~s}, 6 \mathrm{H}, 2 \mathrm{xCH}_{3}\right), 3.39\left(\mathrm{~s}, 4 \mathrm{H}, 2 \mathrm{xCH}_{2}\right), 3.85$ $\left(\mathrm{s}, 6 \mathrm{H}, 2 \mathrm{xOCH}_{3}\right), 4.04\left(\mathrm{~d}, 4 \mathrm{H}, J_{A B}=12.8 \mathrm{~Hz}, 2 \mathrm{xCH}_{2} \mathrm{O}\right), 4.31\left(\mathrm{~d}, 4 \mathrm{H}, J_{A B}=12.8 \mathrm{~Hz}, 2 \mathrm{xCH}_{2} \mathrm{O}\right), 7.75-$ $7.80(\mathrm{~m}, 2 \mathrm{H}, 2 \mathrm{xAr}-\mathrm{H}), 8.17-8.22(\mathrm{~m}, 2 \mathrm{H}, 2 \mathrm{xAr}-\mathrm{H}) ;{ }^{13} \mathrm{C}-\mathrm{NMR} \delta: 21.9\left(2 \mathrm{xCH}_{3}\right), 24.7\left(2 \mathrm{xCH}_{3}\right), 30.5$ $\left(2 \mathrm{xCH}_{2}\right), 61.4\left(2 \mathrm{xCH}_{2} \mathrm{O}\right), 62.3\left(2 \mathrm{xOCH}_{3}\right), 63.9\left(2 \mathrm{xCH}_{2} \mathrm{O}\right), 85.2\left(2 \mathrm{xCNO}_{2}\right), 99.0(2 \mathrm{xC}), 126.0(2 \mathrm{xC})$, $126.7(2 \mathrm{xCH}), 133.9(2 \mathrm{xCH}), 134.0(2 \mathrm{xC}), 137.4(2 \mathrm{xC}), 156.1(2 \mathrm{xC}), 182.2(2 \mathrm{xC}=\mathrm{O})$; Anal. Calcd for $\mathrm{C}_{30} \mathrm{H}_{34} \mathrm{~N}_{2} \mathrm{O}_{12}$ : C, 58.63; H, 5.58; N, 4.56. Found: C, 58.76; H, 5.69; N, 4.38.

General procedure for reactions with substituted sulfinate sodium salt

A solution of substituted sulfinate sodium salt $(3.3 \mathrm{mmol})$ in dimethylsulfoxide $(10 \mathrm{~mL})$ was added dropwise to a solution of dichloride $6(0.20 \mathrm{~g}, 0.55 \mathrm{mmol})$ in dimethylsulfoxide $(6 \mathrm{~mL})$ and stirred under inert atmosphere for $10 \mathrm{~min}$. The reaction mixture was poured into cold water and a precipitate was formed. After filtration, the crude product was recrystallized from the corresponding solvent gave the corresponding bis-S-alkylated product. 
1,4-Dimethoxy-2,3-bis-(toluene-4-sulfonylmethyl)anthraquinone (7g): Yellow solid, mp $281{ }^{\circ} \mathrm{C}$ (ethyl acetate); ${ }^{1} \mathrm{H}-\mathrm{NMR} \delta: 2.42\left(\mathrm{~s}, 6 \mathrm{H}, 2 \mathrm{xCH}_{3}\right), 3.90\left(\mathrm{~s}, 6 \mathrm{H}, 2 \mathrm{xOCH}_{3}\right), 5.07\left(\mathrm{~s}, 4 \mathrm{H}, 2 \mathrm{xCH}_{2} \mathrm{SO}_{2}\right), 7.34(\mathrm{~d}, 4 \mathrm{H}$, $J=8.2 \mathrm{~Hz}, 4 x A r-H), 7.74$ (d, 4H, $J=8.2 \mathrm{~Hz}, 4 x \mathrm{Ar}-\mathrm{H}), 7.76-7.80$ (m, 2H, 2xAr-H), 8.17-8.22 (m, 2H, 2xAr-H); ${ }^{13} \mathrm{C}-\mathrm{NMR} \delta: 21.6\left(2 \mathrm{xCH}_{3}\right), 54.3\left(2 \mathrm{xCH}_{2} \mathrm{SO}_{2}\right), 63.6\left(2 \mathrm{xOCH}_{3}\right), 126.7(2 \mathrm{xCH}), 128.1(4 \mathrm{xCH})$, $130.0(4 \mathrm{xCH}), 132.4(2 \mathrm{xCH}), 133.8(2 \mathrm{xC}), 134.0(2 \mathrm{xC}), 145.3(2 \mathrm{xC}), 156.7(2 \mathrm{xC}), 182.0(2 \mathrm{xC}=\mathrm{O})$; Anal. Calcd for $\mathrm{C}_{32} \mathrm{H}_{28} \mathrm{O}_{8} \mathrm{~S}_{2}$ : C, 63.56; H, 4.67. Found: C, 63.65; H, 4.67.

2,3-Bis(butane-1-sulfonylmethyl)-1,4-dimethoxyanthraquinone (7h): Yellow solid, $\mathrm{mp} \quad 184^{\circ} \mathrm{C}$ (ethanol); ${ }^{1} \mathrm{H}-\mathrm{NMR} \delta: 0.96$ (t, $\left.6 \mathrm{H}, J=6.6 \mathrm{~Hz}, 2 \mathrm{xCH}_{3}\right), 1.43-1.55\left(\mathrm{~m}, 4 \mathrm{H}, 2 \mathrm{xCH}_{2}\right), 1.82-1.94(\mathrm{~m}, 4 \mathrm{H}$, $\left.2 \mathrm{xCH}_{2}\right), 3.04-3.12\left(\mathrm{~m}, 4 \mathrm{H}, 2 \mathrm{xCH}_{2}\right), 3.99\left(\mathrm{~s}, 6 \mathrm{H}, 2 \mathrm{xOCH}_{3}\right), 4.98\left(\mathrm{~s}, 4 \mathrm{H}, 2 \mathrm{xCH}_{2} \mathrm{SO}_{2}\right), 7.77-7.81(\mathrm{~m}, 2 \mathrm{H}$, 2xAr-H), 8.19-8.23 (m, 2H, 2xAr-H); ${ }^{13} \mathrm{C}-\mathrm{NMR} \delta: 13.5\left(2 \mathrm{xCH}_{3}\right), 21.7\left(2 \mathrm{xCH}_{2}\right), 24.0\left(2 \mathrm{xCH}_{2}\right), 50.6$ $\left(2 \mathrm{xCH}_{2} \mathrm{SO}_{2}\right), 53.6\left(2 \mathrm{xCH}_{2} \mathrm{SO}_{2}\right), 63.6\left(2 \mathrm{xOCH}_{3}\right), 126.8(2 \mathrm{xCH}), 127.3(2 \mathrm{xC}), 132.7(2 \mathrm{xC}), 133.5$ $(2 \mathrm{xC}), 134.1(2 \mathrm{xCH}), 156.3(2 \mathrm{xC}), 182.1(2 \mathrm{xC}=\mathrm{O})$; Anal. Calcd for $\mathrm{C}_{26} \mathrm{H}_{32} \mathrm{O}_{8} \mathrm{~S}_{2}$ : C, 58.19; H, 6.01. Found: C, 58.23; H, 5.84.

\section{References and Notes}

1. Lin, A. J.; Cosby, L. A.; Shansky, C. W.; Sartorelli, A. C. Potential bioreductive alkylating agents. 1. Benzoquinone derivatives. J. Med. Chem. 1972, 15, 1247.

2. Moore, H. W. Bioactivation as a model for drug design bioreductive alkylation. Science 1977, $197,527$.

3. Arcamone, F. Properties of antitumor anthracyclines and new developments in their application: Cain Memorial Award Lecture. Cancer Res. 1985, 45, 5995.

4. Vanelle, P.; Terme, T.; Giraud, L.; Crozet, M. P. Progress in electron transfer reactions of new quinone bioreductive alkylating agents. Recent Adv. Devel. Org. Chem. 2000, 4, 1.

5. Terme, T.; Crozet, M. P.; Maldonado, J.; Vanelle, P. In Electron Transfer Reactions in Organic Synthesis, Vanelle, P., Ed.; Research Signpost, Trivandrum, 2002, 1.

6. Kerdesky, F. A. J.; Ardecky, R. J.; Lakshmikantham, M. V.; Cava, M. P. Simple o-quinodimethane route to $( \pm)$-4-demethoxydaunomycinone. J. Am. Chem. Soc. 1981, 103, 1992.

7. Terme, T.; Crozet, M. P.; Giraud, L.; Vanelle, P. Original annulation in anthraquinone series: synthesis of new 2,3-dialkylnaphthacene-5,12-diones. Tetrahedron 2000, 56, 1097.

8. Liu, L. K.; Chi, Y.; Jen, K.-Y. Copper-catalyzed additions of sulfonyl iodides to simple and cyclic alkenes. J. Org. Chem. 1980, 45, 406.

9. Gilbert, K. E.; Borden, W. T. Peracid oxidation of aliphatic amines: general synthesis of nitroalkanes. J. Org. Chem. 1979, 44, 659.

10. Linden, G. B.; Gold, M. H. Preparation of 2- and 5-substituted 1,3-dioxanes. J. Org. Chem. 1956, 21,1175 .

Samples Availability: Available from the authors.

(C) 2005 by MDPI (http://www.mdpi.org). Reproduction is permitted for noncommercial purposes. 